\title{
Durchschleusen von Trübeströmen durch einen alpinen Speicher
}

\author{
Venting of turbidity currents through an Alpine reservoir
}

\section{J. SCHNEIDER, W. DOBLER und H. BADURA}

\section{Kurzfassung/Summary}

Der hier vorgestellte Beitrag befasst sich mit Naturmessungen und numerischen Berechnungen von Durchschleusevorgängen von Trübeströmen am Sölkspeicher in der Obersteiermark. Es werden an insgesamt 5 Messstellen hydrologische/physikalische Parameter wie Abfluss, Fließgeschwindigkeit, Trübe, Leitfähigkeit sowie die Wassertemperatur erfasst und das zeitliche Auftreten der einzelnen Parameter an den unterschiedlichen Messstellen registriert.

Numerische Untersuchungen verschiedenster Art werden im Rahmen des Projektes realisiert. So wird versucht, mittels Large Eddie Simulationen das Auftreten von Trübeströmen $\mathrm{zu}$ simulieren und die Anwendbarkeit dieser Methode zu überprüfen. Aufbauend auf bereits früher durchgeführte einfache 1D Untersuchungen werden die Strömungsvorgänge mittels 3-dimensionalen Berechnungen naturnah nachgebildet. Es zeigt sich, dass die einphasigen Simulationen (nur Wasser) ähnliche Strömungsmuster als die Naturbeobachtungen aufweisen. Sowohl die Fließgeschwindigkeiten als auch die Fließrichtungen stimmen sehr gut überein. Erste zweiphasige Berechnungen (trübes Wasser tritt in klares Wasser ein) lassen auf das Auftreten von Trübeströmen im Speicher schließen und sollen in weiterer Folge noch näher betrachtet werden.

Venting processes of turbidity currents have been studied in the Sölk reservoir in upper Styria using field measurements and numericalanalyses. Hydrological/physical parame- ters such as flow, velocity, turbidity, conductivity and water temperature are measured at 5 stations, with the timed occurrence of each parameter being registered at the various stations.

The current study project includes diverse numerical analyses. Large Eddy Simulations are used in an attempt to simulate the occurrence of turbidity currents and check the practicability of this method. Based on earlier simple $1 D$ studies, near-natural models of flow processes have been established using $3 D$ analyses. The results have shown that single-phase simulations (water only) give flow patterns similar to those observed in the field. Both flow velocities and flow directions show very satisfactory agreement. First two-phase analyses (where turbid water enters into clear water) suggest that turbidity currents do occur in the reservoir; they will be the subject of further study.

\section{Einleitung}

Österreichweit werden ca. 2/3 der elektrischen Energie mittels Wasserkraft erzeugt. Durch die zunehmende Energiekonsumation sinkt dieser Anteil kontinuierlich und es besteht das Bestreben, dass neue Anlagen errichtet bzw. bestehende Anlagen saniert und optimiert werden. Von einem gegenwärtig in Österreich bestehenden Jahresarbeitsvermögen von $38 \mathrm{TWh}$ ausgehend wird eine Zunahme um 7 TWh bis zum Jahr 2020 angestrebt (KollerKreimel, 2008).

Wasserkraftwerke können einerseits durch kleinere Stauhaltungen bei Niederdruckanlagen üblicherweise an Flüssen gekennzeichnet sein, andererseits durch große Speicher im inneralpinen Raum, wie sie bei Hochdruckanlagen mit großen Fallhöhen zum Einsatz kommen. All diese Anlagen bilden künstliche Barrieren in den jeweiligen Fließgewässern. Dies hat zur Folge, dass die natürlichen Wanderwege aquatischer Lebewesen und auch der Sedimenttransport unterbrochen bzw. stark eingeschränkt sein können.

Die vorliegende Arbeit stellt Ergebnisse vor, welche im Rahmen des Forschungsprojektes Einfluss von Trübeströmen auf die Speicherverlandung - Durchschleusen als Alternative (ETS) gewonnen wurden. Da das Projekt noch nicht abgeschlossen ist und sowohl die Naturmessungen als auch die numerischen Berechnungen noch im Bearbeitung sind, können hier nur erste Ergebnisse präsentiert werden, welche jedoch bereits einen sehr guten Eindruck in die Möglichkeit des Durchschleusens von Trübeströmen geben.

\section{Problemstellung}

ÖWAV (1998) unterscheidet folgende drei Kategorien von Stauanlagen bezüglich Feststoffproblematik:

Kategorie A: Der Feststoffhaushalt ist in der Betriebsvorschrift geregelt (z. B. bei Wasserfassungen) und wird als Routinemaßnahme beherrscht. Der Speicherinhalt ist meist sehr gering und die Verweildauer des Wassers so kurz, dass die Feinpartikel gar nicht zum Absetzen gelangen können. Der abgelagerte Grobanteil kann meist durch Spülung oder Baggerung entfernt werden. 


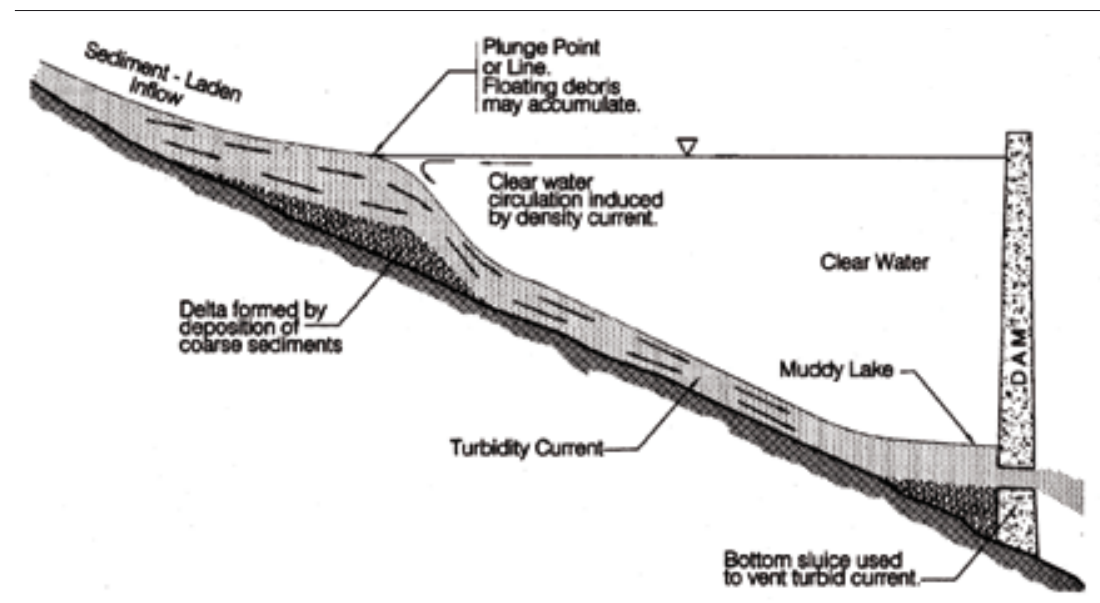

Abb. 1: Ausbildung eines Dichtestromes und Abfluss durch den Grundablass (Morris und Fan, 1998)

Kategorie B: Der Feststoffhaushalt stellt Verlandungsprobleme dar, welche durch Maßnahmen zu lösen sind. Bei diesen Speichern ist ein Sedimentaustrag mittelfristig erforderlich, was durch den quantitativ hohen Feinstoffanteil bei Spülungen im Unterlauf zu ökologischen Problemen führen kann.

Kategorie C: Der Feststoffhaushalt ist nicht oder nur langfristig - auf Jahrzehnte - relevant (z. B. große Speicher und Pumpspeicherbecken). Die große Verweildauer des Wassers im Speicher (Verhältnis kleiner Zufluss zu großem Volumen) bewirkt die Sedimentation der Feststoffe, was jedoch durch das Vorhandensein großer Toträume keine Probleme darstellt.

Der im Rahmen dieses Forschungsprojektes untersuchte Sölkspeicher kann durch seine Geometrie der Kategorie B zugeordnet werden. Die Notwendigkeit der Sedimentbewirtschaftung des Speichers sowie vorhergehende Untersuchungen des gesamten Einzugsgebietes (Habersack et al., 2002) stellten die Grundlage für die Auswahl dieses Speichers dar.

In den Speicher Großsölk gelangen einerseits Feststoffe aus dem natürlichen Einzugsgebiet, andererseits bestehen auch zwei Beileitungen aus drei benachbarten Einzugsgebieten, welche Feinmaterial in den Speicher eintragen. Das Geschiebe wird in einem extra dafür vorgesehenen Geschiebeauffangraum, der durch eine Vorsperre vom Speicher abgetrennt ist, abgelagert und je nach Bedarf mechanisch geräumt. Das im Speicher abgelagerte Feinmaterial wurde bisher durch drei Stauraumspülungen wieder in den Unterlieger transferiert. Während der mehrtägigen Spülungen kommt es zu einer Unterbrechung der Energieproduktion mit einer damit verbundenen Kostenentwicklung. Zusätzlich ist, wie bereits erwähnt wurde, eine erhebliche Schwebstoffbelastung der Abschnitte unterhalb der Sperre bis hin zur unterliegenden Enns zu erwarten.

\section{Zielsetzungen}

Das Durch- bzw. Vorbeischleusen von Trübeströmen durch einen oder an einem Speicher ist nicht zu verwechseln mit Spülungen von Speichern. Es wird zwar bei beiden Bewirtschaftungsmethoden der Grundablass geöffnet, jedoch wirken unterschiedliche Mechanismen. Während bei einer Spülung der Stauraum üblicherweise entleert wird und damit die Schleppkräfte das abgelagerte Sediment wieder remobilisieren können, wird beim Durchschleusen der Speicherspiegel nicht abgesenkt. Es soll sich das in den Speicher einfließende schwerere, mit Schwebstoffen beladene Wasser, entlang des Talweges bis zur Sperre fortbewegen, und dort durch den Grundablass in das Unterwasser abgegeben werden. Somit ist es möglich, Verlandungsprozesse zu unterbinden bzw. zumindest zu verringern. Eine nähere Betrachtung der Wirtschaftlichkeit dieser Durchschleusevorgänge wird in Putz (2007) untersucht. Des Weiteren kann davon ausgegangen werden, dass das durch den Speicher geschleuste Wasser üblicherweise keine signifikant höheren Sedimentkonzentrationen aufweist, als während eines natürlichen Hochwasserereignisses. Natürlich können im Speicher Vermischungsprozesse mit klarerem Wasser und auch Remobilisierungsprozesse mit einhergehenden Konzentrationserhöhungen auftreten. Inwieweit dies beim Sölkspeicher der Fall ist soll u.a. in diesem Forschungsprojekt geklärt werden. In Abbildung 1 ist ein idealisierter Durchschleusevorgang durch einen Speicher dargestellt.

In der vorliegenden Arbeit sollen die ersten Ergebnisse der Naturmessungen und der durchgeführten numerischen Berechnungen vorgestellt werden. Es können jedoch noch keine abschließenden Resultate präsentiert werden, da die Messkampagne im Jahre 2008 noch fortgesetzt wird und die damit verbundenen Resultate noch nicht vorliegen.

\section{Grundlagen und Methoden}

\subsection{Theorie der Dichteströme}

In Speichern oder Seen kommt es immer wieder zu Schichtungen des Wasserkörpers. Diese können durch Temperaturunterschiede, gelöste Stoffe, in Schwebe gehaltene Stoffe oder durch eine Kombination der oben genannten Möglichkeiten verursacht werden. Dichteunterschiede können jedoch auch zwischen dem Zufluss in ein ruhendes Gewässer und ebendiesem auftreten. In Abbildung 2 sind Möglich- 


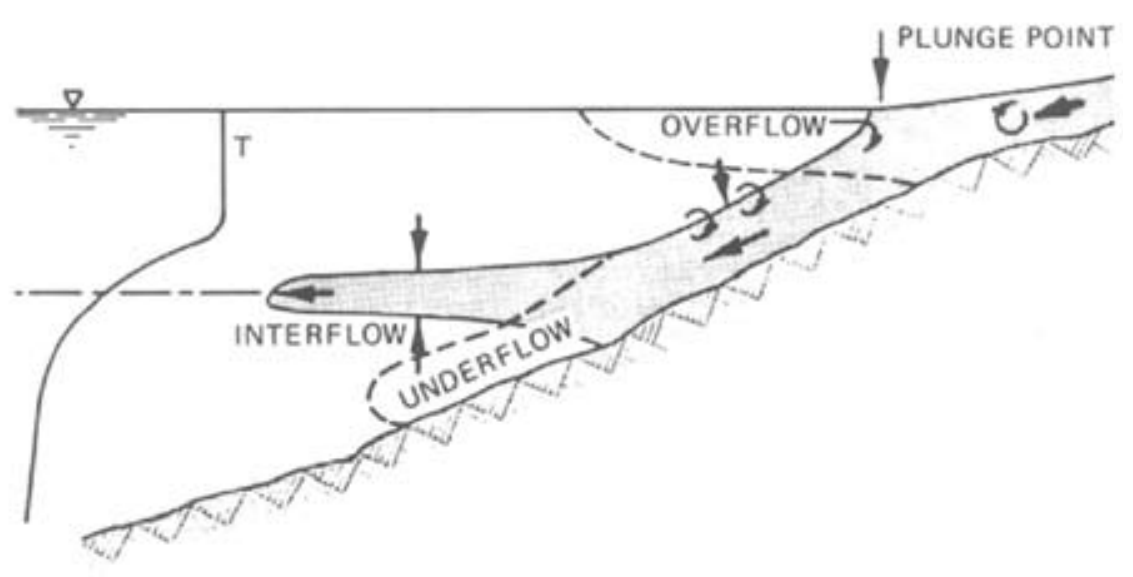

Abb. 2: Abtauchpunkt und mögliche Dichteströme (Ford und Johnson, 1983)

keiten der Dichtestromausbildung dargestellt. Je nachdem ob der Zufluss leichter, schwerer oder ob eine spezielle Schichtung im See vorherrscht, können underflows, interflows oder overflows entstehen.

Der Begriff „Dichtestrom“ kann als Überbegriff sowohl für temperaturabhängige als auch für schwebstoffabhängige Strömungsformen verstanden werden. Wenn Schwebstoff im Spiel ist, bezeichnet man diese Art des Abflusses als „Trübestrom“ (engl.: turbidity current).

\subsection{Naturmessungen}

Einen Schwerpunkt dieses Forschungsprojektes bilden die Messungen am Sölkspeicher. In Abbildung 3 ist eine Übersicht aller Messstationen sowie der dort erfassten Parameter dargestellt. Oberwasserseitig des Zuflusses Großsölkbach in den Speicher befindet sich die Messstation 1, bei der der Zufluss, die Trübe und Temperatur sowie die Leitfähigkeit gemessen werden. Im ersten Drittel des Speichers (Messstelle 2) sind Messsonden an einem vertikalen Seil direkt über der tiefsten Stelle des Querprofils montiert, welche die Geschwindigkeit sowie die Temperatur, Leitfähigkeit und Trübe in zwei unterschiedlichen Wassertiefen erfassen. Bei der Geschwindigkeitssonde handelt es sich um einen Acoustic Doppler Current Meter
„Argonaut XR“ der Firma Sontek, der in verschiedenen Wassertiefen die Fließgeschwindigkeiten in drei Dimensionen erfassen kann.

Direkt am Grundablassbauwerk wurde ein 8 Meter auskragender Stahlträger montiert, an dessen Ende sich die gleichen Messgeräte wie an der Messstelle 2 befinden (Messstelle 3).

Die erfassten Daten an diesen drei Messstationen werden in einem Datenlogger gespeichert und via GSM fernübertragen.

Einige hundert Meter unterhalb des Grundablassauslaufbauwerkes befindet sich die Messstation 4, welche im Falle des Öffnens des Grundablasses die Trübe und Temperatur erfasst. Die Messstation 5 schließlich speichert die Trübewerte direkt im Bereich des Turbinenauslaufes beim Krafthaus. Eine detaillierte Beschreibung der Messanordnung ist in Troy (2006), Wachter (2008) und Badura \& Schneider (2008) nachzulesen.

\subsection{Numerische Strömungssimu- lation}

Im Rahmen des Forschungsprojektes ETS wurden einerseits numerische Voruntersuchungen allgemeiner Natur (Dobler, 2005), andererseits spezifische, auf den Sölkspeicher abgestimmte Berechnungen durchgeführt.

\subsubsection{Voruntersuchung}

Für eine erste Voruntersuchung der Dichteströmung im Sölkspeicher wird das hauseigene Programm der Universität von Iowa (IIHRHydroscience \& Engineering, USA, Supervisor: G. Constantinescu) verwendet. Dabei handelt es sich um eine Large Eddy Simulation (LES) welche die Navier-Stokes Gleichungen in dimensionsloser Form mittels finiten VolumenVerfahren und einem kartesischen Netz löst. Der Vorteil einer LES ist die Möglichkeit, dass in einer Strömungssimulation die großskaligen Wirbelstrukturen (Eddies) ohne Turbulenzmodell aufgelöst, und nur die kleinen Wirbelstrukturen mittels Turbulenzmodell gelöst werden müssen. Dies ist sozusagen eine genauere Beschreibung der Strömungsvorgänge als mit einer herkömmlichen 3-D Simulation möglich ist. Das Ergebnis dieser Strömungsberechnung ist somit einer Standard k-e Berechnung weit überlegen und nur die enormen Rechenkapazitäten einer LES sind der Grund, dass dieses Werkzeug hauptsächlich nur in der Forschung eingesetzt werden kann.

\subsubsection{Numerische Berechnung des Sölkspeichers}

Erste eindimensionale, numerische Berechnungen wurden bereits im Rahmen eines an der Universität für Bodenkultur Wien durchgeführten Forschungsprojektes publiziert (Schneider, 2002; Schneider, 2004; Habersack et al., 2002). Eine detaillierte 3-dimensionale numerische Strömungsberechnung wurde und wird im Rahmen des Projektes ETS noch durchgeführt. Die ersten Simulationen erfolgten noch einphasig (ohne Sediment) und die daraus gewonnenen Ergebnisse wurden bereits mit Naturmessungen verglichen. Die anschließenden zweiphasigen Kalkulationen sind noch nicht abgeschlossen und daher können momentan nur vorläufige Ergebnisse publiziert werden. Die 


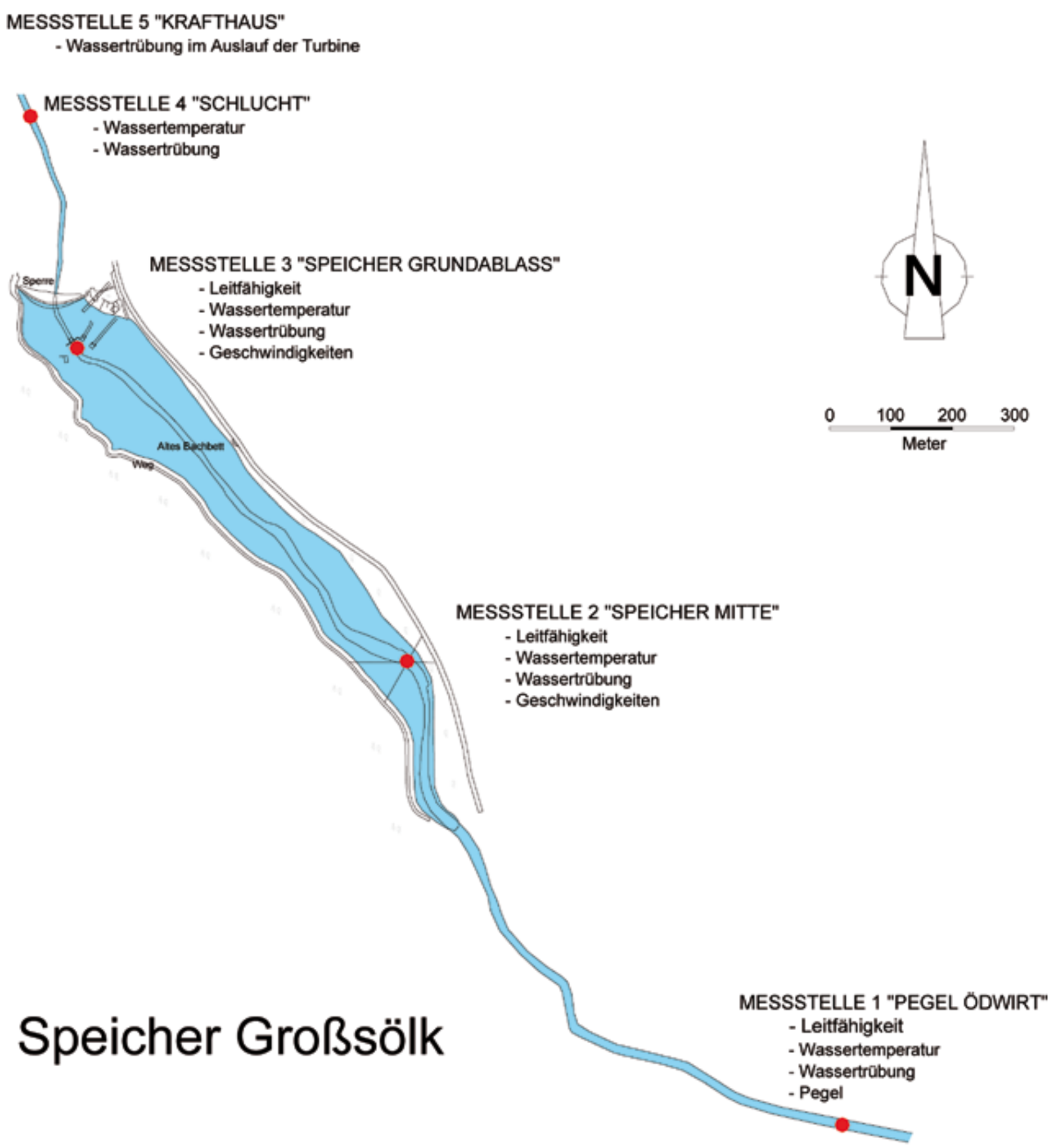

Abb. 3: Überblick der Messstellen am Speicher Großsölk

Strömungsberechnung für das ganze Staubecken werden mit einem kommerziellen Softwarepaket (Fluent 6.2, Ansys) durchgeführt.

\section{Ergebnisse und Vergleiche}

In diesem Kapitel werden Ergebnisse der Naturmessungen sowie erste Resultate der numerischen Berechnungen und deren Vergleich mit den Naturmessungen dargestellt.

\subsection{Naturbeobachtungen}

Es wurden mehrere kleinere Ereignisse beobachtet, bei denen erhöhte
Abflüsse und damit verbundene höhere Sedimentkonzentrationen aufgetreten sind. Beispielhaft soll das Ereignis vom 9.7. auf den 11.7. 2007 herangezogenwerden. Dieses Ereignis war das größte bisher beobachtete Abflussereignis während des Projektszeitraumes. Am 10.7. 2007 wurde der Grundablass geöffnet, da 
bereits die Hochwasserentlastung angesprungen war und somit das überschüssige Wasser teils auch durch diesen abgeführt werden konnte. Genaugenommen handelt es sich bei diesem Ereignis um zwei Ereignisse, wobei die erste Spitze am 9.7. um 09:10 Uhr und die zweite, größere $\left(27,17 \mathrm{~m}^{3} / \mathrm{s}\right)$ um 21:40 Uhr erreicht wurden. In Abbildung 4 ist der Verlauf der Trübung bei den einzelnen Trübesonden dargstellt. Es zeigte sich während des Ereignisses, dass zeitgleich mit der Abflussspitze auch die höchsten Trübewerte bei der Station 1 aufgetreten sind. Die Höchstwerte der Trübe wurden bei der Station 2 ca. 40 Minuten beim kleineren ersten Ereignis und 50 Minuten später bei der nachfolgenden größeren Welle registriert. Bei der Messstelle 3 am Grundablass wurde das erste Ereignis gar nicht erfasst, die zweite Spitze ca. 2 Stunden nach den höchsten registrierten Werten bei Station 2.

Interessant ist auch der Trübeverlauf bei der Messstation 5, welcher dadurch gekennzeichnet ist, dass die Werte einige Stunden nach der ersten Hochwasserwelle leicht ansteigen. Das lässt den Schluss zu, dass trübes Wasser zwar nicht beim Grundablass registriert, aber über die Triebwasserentnahme aus dem Stausee abgeführt wurde. Ein starker Anstieg der Trübe unterwasserseitig der Turbine ist jedoch bei der größeren Hochwasserwelle deutlich erkennbar. Augenscheinlich ähnelt der Trübeverlauf jenem bei der Messstelle 3, wobei eine zeitliche Verzögerung zu beobachten ist, welche auf die Länge des Triebwasserweges zurückzuführen ist. Des Weiteren erreicht die Trübe bei Station 5 höhere Werte, was darauf schließen lässt, dass die größere Menge an Sediment über den Triebwasserweg abgeführt wurde.

Obwohldie Messdaten derMessstelle 4 nur begrenzt vorhanden sind, ist zu erkennen, dass aufgrund des geöffneten Grundablasses am 10.7. von ca. $14: 00$ Uhr bis 19:00

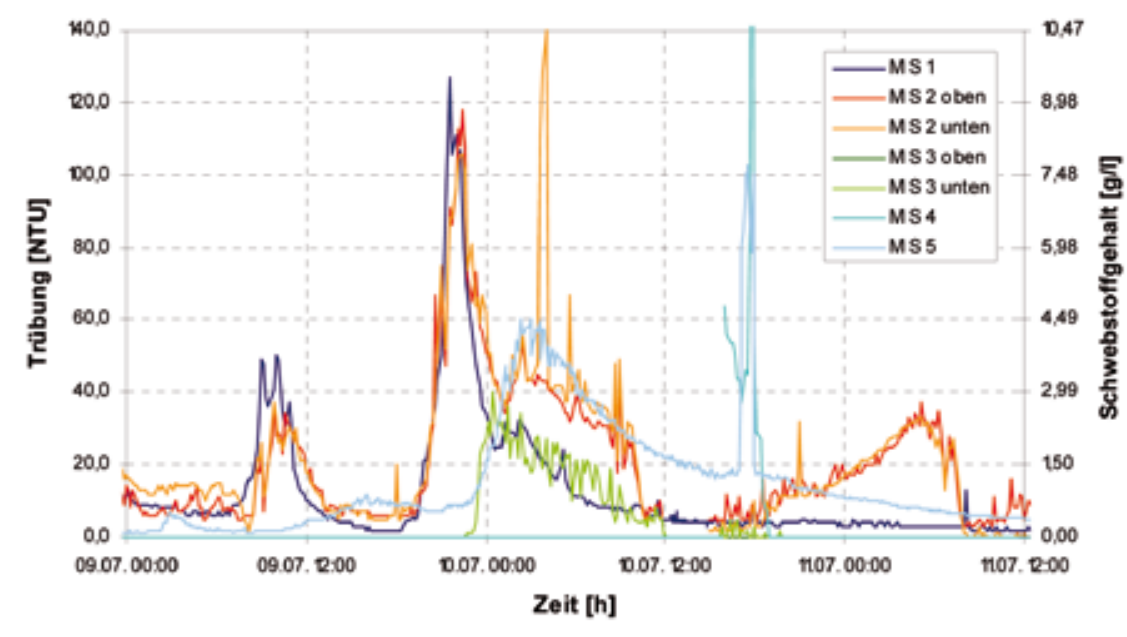

Abb. 4: Trübwerte bei den Messstationen 1 bis 4 während des Ereignisses vom 9.7. bis 11.7. 2007 (Wachter, 2008)

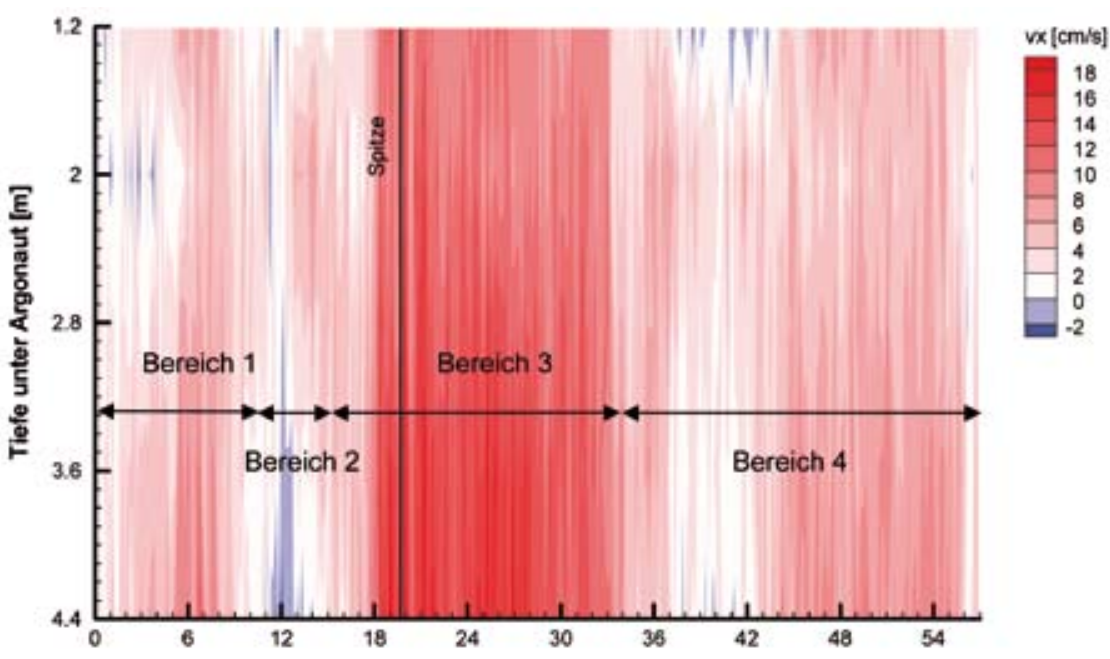

Abb. 5: Fließgeschwindigkeiten an der Messstelle 2, vx, 9.7.2007 03:00Uhr bis 11.7.2007 12:00Uhr (Wachter, 2008)

Uhr die Trübung im Bereich der Schlucht stark zu steigen beginnt. In weiterer Folge ist diese Erhöhung der Trübewerte auch bei der Messstelle $5 \mathrm{zu}$ erkennen. Da während dieser Zeit (17:00 Uhr bis 18:00 Uhr) die Turbine abgestellt war, ist diese Erhöhung auf ein Durchmischen des Wassers beim Turbinenauslauf mit dem Wasser des Sölkbaches zu erklären. Die zeitliche Verzögerung der Erfassung der Trübewerte an den einzelnen Messstationen konnte die durchfließende Welle während dieses Ereignisses sehr gut beschreiben.

In Abbildung 5 sind die Geschwindigkeiten in Hauptfließrich- tung $(\mathrm{x}$-Richtung $=$ Richtung altes Flussbett) im sohlnahen Bereich bei der Messstelle 2 dargstellt. Man erkennt eindeutig unterschiedliche Bereiche, wobei eine vertikale Geschwindigkeitsverteilung (Ausnahme evtl. Bereich 2) nicht erkennbar ist. Der erste Bereich kennzeichnet die Zeitspanne der ersten kleinen Hochwasserwelle. Hier fließt das Wasser eindeutig, wenn auch langsam, in Richtung Sperre. Die Geschwindigkeiten, die während des Zeitraumes des Bereiches 2 erfasst wurden, waren ebenfalls sehr gering, zeigten jedoch v. a. in tieferen Schichten in Richtung zur Stauwurzel. Diese Rückströmungen 


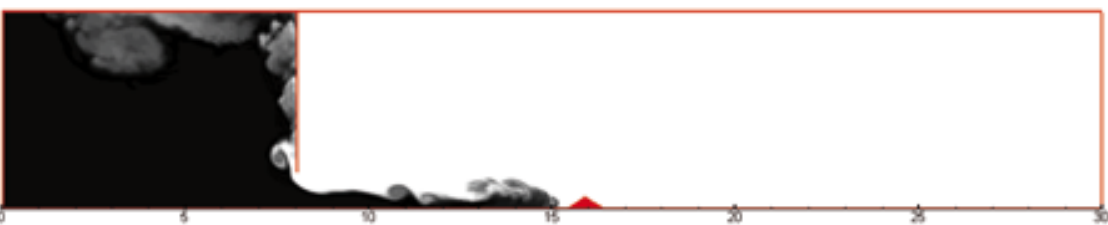

Abb. 6: Geometrie für die LES, Teilergebnis der LES bei $t=2[s]$ und $R e=2382$ (Dobler, 2006)

konnten auch mit den Temperatursonden verifiziert werden. Der dritte Bereich beschreibt den Zeitraum der größeren Welle und die Fließrichtung unterhalb der Geschwindigkeitssonde war eindeutig in Richtung Sperre erfasst worden. Der Bereich 4 schließlich zeigt die Geschwindigkeiten nach den beiden Ereignissen. Die Geschwindigkeiten waren sehr gering. Der neuerliche Wiederanstieg der Trübe an der Messstelle 2 am Nachmittag des 10.7. (Abbildung 4) ist jedoch durch die Geschwindigkeitserfassung nicht ganz nachvollziehbar. Man kann wahrscheinlich davon ausgehen, dass sich in diesem Zeitraum großräumige Wirbelstrukturen mit indifferenten Strömungsmustern ausgebildet haben.

\subsection{Numerische Strömungs- simulationen}

\subsubsection{Voruntersuchung}

Die Geometrie für die Voruntersuchung mittels LES besteht aus einem einfachen Rechteckquerschnitt sowie einem am Boden befindlichen Damm. Das dichtere Medium ist durch eine Barriere vom leichteren Medium getrennt und nur in Bodennähe ist eine Öffnung vorhanden, so dass es zu einem Austausch zwischen den zwei Medien kommen kann. (siehe Abbildung 6). Der prinzipielle Vorgang ist der, dass aufgrund der Gravitation das dichtere Medium durch die Öffnung in der Barriere in das leichtere Medium eindringt, eine Dichteströmung bildet und schließlich auf den Damm trifft und darüber strömt.

Mehrere Simulation mit unterschiedlicher Geometrie (Höhe des Modells, der Öffnung und des Dammes) wurden durchgerechnet, um die geometrischen Größen des Dichtestroms zu erlangen. Die Kalibrierung erfolgte mittels Laborversuchen (Oehy, 2003, Lane - Serff et al., 1995) und zeigte eine gute Übereinstimmung mit der LES. Weiters wurde die Sohlschubspannung ermittelt, da die Netzauflösung an der Sohle bis in die viskose Unterschicht reicht und somit das newtonsche Gesetz gilt:

$$
\tau=\eta \frac{d u}{d y}
$$

wobei $\tau$ die Sohlschubspannung, $\eta$ die dynamische Zähigkeit und du/ dy den Geschwindigkeitsgradienten darstellt. Alle Zahlenwerte sind in dimensionsloser Form angegeben. Ein Beispiel für die dimensionslose
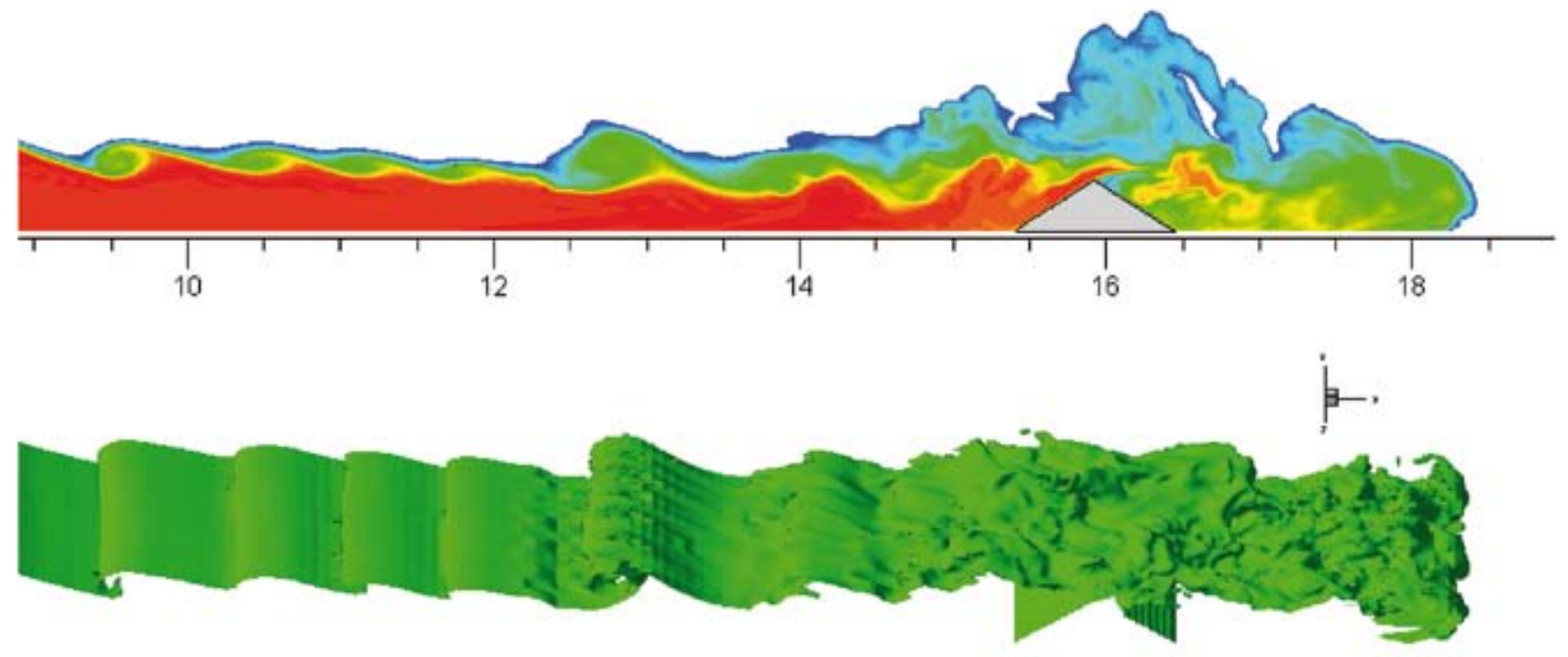

tau
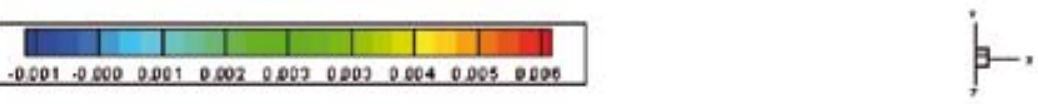

Abb. 7: Von oben nach unten: Schnitt durch die Symmetrieebene des Dichtestroms, 3D Ansicht sowie dimensionslose Sohlschubspannung (Dobler, 2006) 


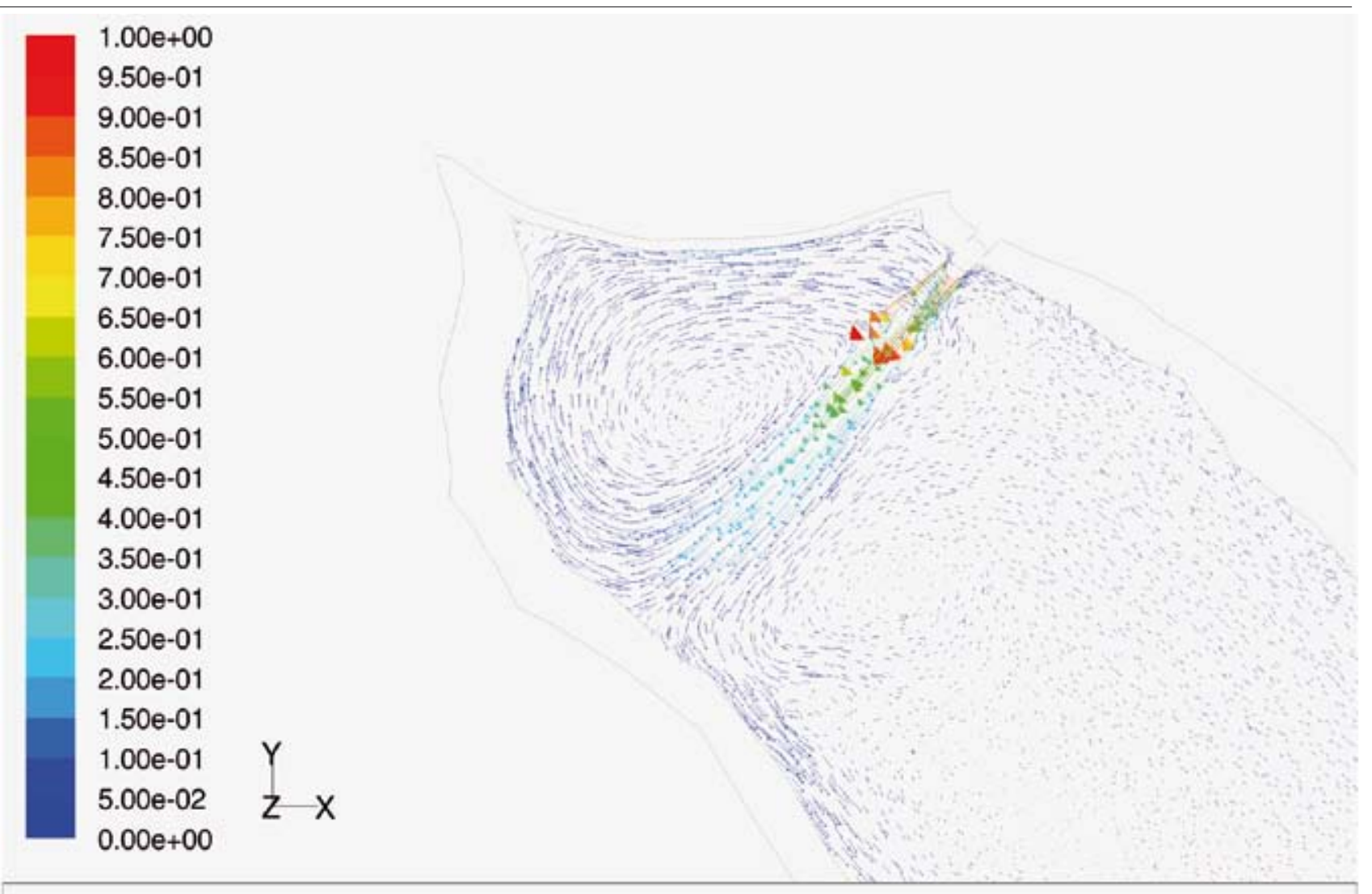

Velocity Vectors Colored By Velocity Magnitude $(\mathrm{m} / \mathrm{s})$ (Time $=9.5990 \mathrm{e}+04)$

Apr 03, 2007 FLUENT 6.2 (3d, segregated, ske, unsteady)

Abb. 8: Horizontalschnitt bei 894 m ü.A. durch den Sölkspeicher, Geschwindigkeitsvektoren

Sohlschubspannung, nachdem der Kopf des Dichtestroms den Damm passiert, ist in Abbildung 7 ersichtlich.

\subsubsection{Numerische Berechnung des Sölkspeichers}

Die Geometrie des gesamten Staubeckens wurde mittels Tetraedern sowie Hexaedern vernetzt und somit die komplizierte Struktur der Sohle und die Ein- und Ausläufe detailgenau modelliert. Um das Verhalten der zwei Phasen (Reinwasser im Becken sowie mit Sediment beladene Dichteströmung) in Fluent zu modellieren, wird für die Zweiphasenrechnung die „Euler-Euler-Methode“ verwendet. Dadurch wird das Durchmischen der Phasen mitmodelliert und pro Phase die Navier-Stokes Gleichung sowie die Kontinuitätsgleichung gelöst.

Die Randbedingungen für die einphasige Modellierung waren wie folgt definiert: Zufluss Großsölkbach $13,0 \mathrm{~m}^{3} / \mathrm{s}$, Beileitung Donnersbach 10,0 $\mathrm{m}^{3} / \mathrm{s}$, Beileitung Kleinsölk 7,0 $\mathrm{m}^{3} / \mathrm{s}$, Abfluss Triebwasserstollen $30,0 \mathrm{~m}^{3} / \mathrm{s}$. Der Grundablass wurde geschlossen angenommen, da nur mit Klarwasser gerechnet wurde. In Abbildung 8 sind beispielhaft die Geschwindigkeitsvektoren, hervorgerufen durch das Einlaufbauwerk der Beileitung Donnersbach in einer Wassertiefe von $894 \mathrm{~m}$ ü. A. (8 Meter unter Stauziel) dargestellt, wie sie bei einer einphasigen Rechnung auftreten.

Für die numerische zweiphasige Berechnung, welche hier beispielhaft dargestellt ist, ist folgendes Szenario festgelegt worden:
Zufluss Großsölkbach 13,82 $\mathrm{m}^{3} / \mathrm{s}$, Zufluss Beileitung Kleinsölkbach $11,62 \mathrm{~m}^{3} / \mathrm{s}$, Zufluss Beileitung Donnersbach $8,50 \mathrm{~m}^{3} / \mathrm{s}$, Abfluss Triebwasserstollen $\quad 25,46 \mathrm{~m}^{3} / \mathrm{s}$, Abfluss Grundablass $8,49 \mathrm{~m}^{3} / \mathrm{s}$. Es zeigte sich bei dieser Rechnung, dass mit Schwebstoff beladenes Wasser aus dem Großsölkbach in den Stauraum eindringt und sich ein Dichtestrom ausbildet. Die numerische Berechnung zeigt somit, dass das mit Schwebstoff beladene Wasser durchaus die Form einer Dichteströmung annimmt. Abbildung 9 veranschaulicht die Trübewolke nach einer Stunde in Natur. Die Werte zwischen 0 und 1 beschreiben normierte Phasen, wobei 0 die leichte $\left[998 \mathrm{~kg} / \mathrm{m}^{3}\right.$ ] und 1 die schwere Phase $[1010 \mathrm{~kg} /$ $\mathrm{m}^{3}$ ] darstellt. Werte unter 0,1 sind in Abbildung 9 ausgeblendet. 


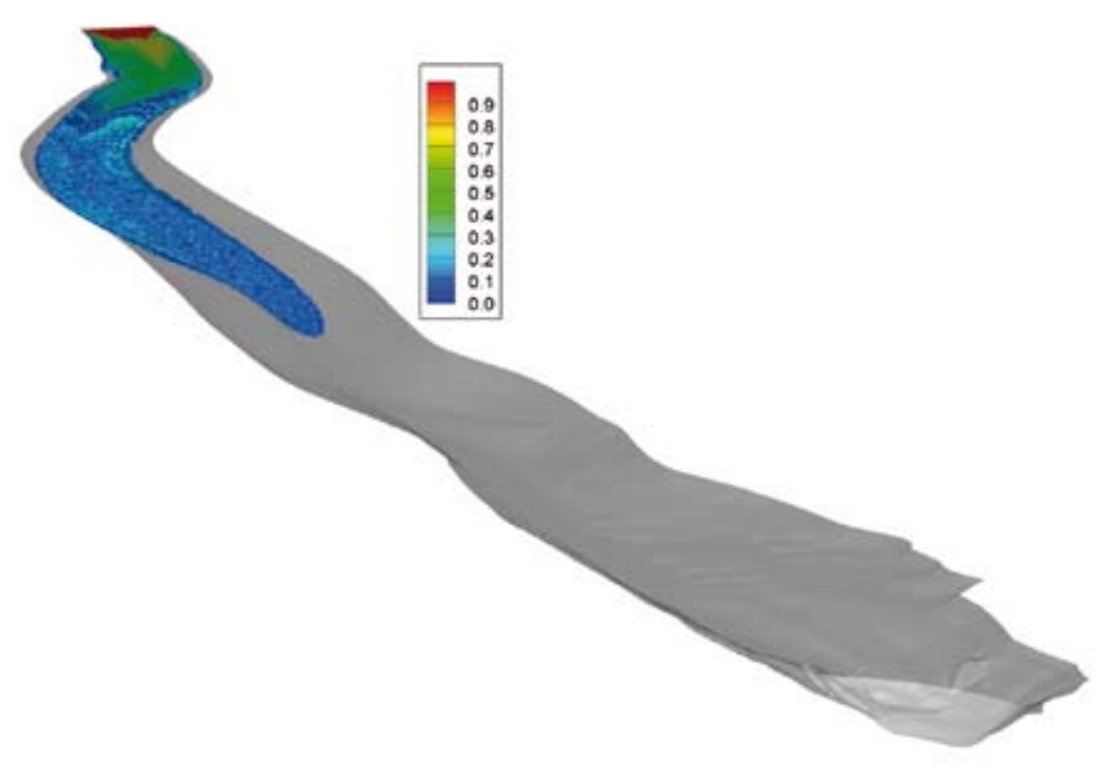

Abb. 9: Trübestrom im Sölkspeicher nach ca. 1 Stunde

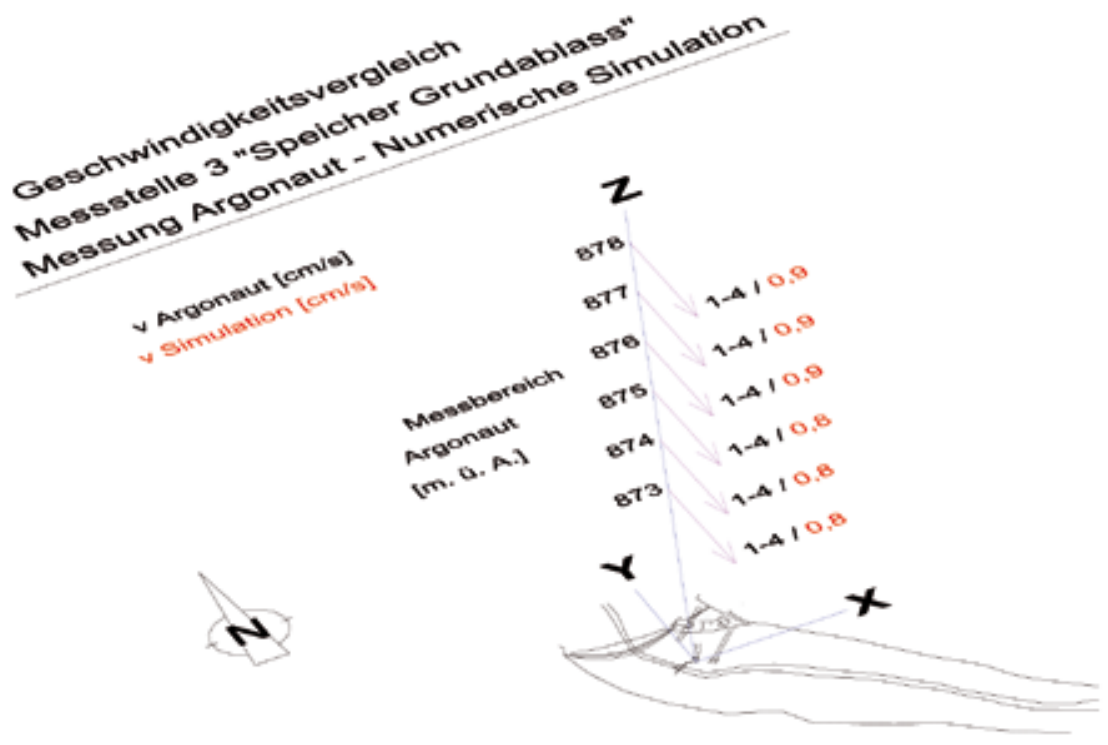

Abb. 10: Vergleich der Fließgeschwindigkeiten und Fließrichtung an der Messstelle 3 (Troy, 2006)

\subsubsection{Vergleich der numerischen Berechnung mit Naturmes- sungen}

Wie bereits erwähnt kann eine Kalibrierung der Numerik zum jetzigen Zeitpunkt nur für die einphasigen Berechnungen vorgestellt werden. In Abbildung 10 wird der Vergleich der Simulation mit den an der Messstelle 3 registrierten Geschwindigkeitsgrößen dargestellt. Die gemes-

\section{Zusammenfassung}

Der vorliegende Beitrag befasst sich mit Ergebnissen eines noch laufenden und somit sehr aktuellen Forschungsprojektes, welches den Versuch unternimmt, Trübeströme, hervorgerufen durch schweres, mit Schwebstoffen beladenes, in einen Stausee einfließendes Wasser durch geeignete Betriebsführung durch den Speicher zu schleusen. Beim untersuchten Speicher handelt es sich um den in der Obersteiermark befindlichen Sölkspeicher. Das Projekt läuft seit 2006 und endet im Dezember 2008. Die bisher höchste beobachtete Hochwasserspitze während der Projektdauer lag bei $27,17 \mathrm{~m}^{3} / \mathrm{s}$. Das $\mathrm{HQ}_{1}$ für den Großsölkbach ist mit $42 \mathrm{~m}^{3} / \mathrm{s}$ definiert. Daher zeigt sich schon das Problem, dass bisher kein großes Hochwasserereignis mit hohen Schwebstoffkonzentrationen erfasst wurde. Nichtsdestotrotz konnten bereits mehrere kleine Ereignisse beobachtet werden, bei denen die Trübewolken bis zum Grundablass erfasst wurde.

Die numerischen Voruntersuchungen mittels LES zeigten, dass Trübeströme mit dieser Methode bereits sehr gut abgebildet werden können. Die äußerst naturähnliche Beschreibung der Strömungsvorgänge mittels LES kann jedoch zum jetzigen Zeitpunkt nur bei sehr einfachen Geometrien (Rechteckquerschnitt, keine Sohlneigung) aufgrund der enormen Rechenkapazitäten realisiert werden. In naher Zukunft kann jedoch damit gerechnet werden, dass diese $\mathrm{Si}$ mulationen auch für kompliziertere Geometrien angewendet werden.

Die numerischen einphasigen Berechnungen mittels 3D Numerik stimmten sehr gut mit den Naturmessungen überein, wobei die Geschwindigkeiten und die Fließrichtung richtig erfasst wurden. Die ersten Resultate der zweiphasigen Berechnungen zeigen den Ansatz einer Trübestromausbildung und 
werden in nächster Zukunft weiter untersucht. Das kalibrierte Modell soll bis zum Ende des Projektes für Variantenuntersuchungen herangezogen werden.

\section{Danksagung}

Das Forschungsprojekt Einfluss von Trübeströmen auf die Speicherverlandung - Durchschleusen als Alternative wird seit 2006 gemeinsam mit der VERBUND - Austrian Hydro Power AG (AHP) durchgeführt. Die
Österreichische Forschungsgesellschaft mbH (FFG) fördert das Projekt, indem sie $60 \%$ der Projektkosten übernimmt, der Rest wird von der AHP bestritten.

\section{Korrespondenz:}

Dipl.-Ing. Dr. Josef Schneider, Dipl.-Ing. Wolfgang Dobler, Institut für Wasserbau und Wasserwirtschaft, Technische Universität Graz

Dipl.-Ing. Dr. Hannes Badura, VERBUND - Austrian Hydropower AG

\section{LITERATUR}

BADURA H, SCHNEIDER J (2008): Durchleiten von Trübeströmen am Beispiel des Speichers Großsölk, Neue Anforderungen an den Wasserbau, Internationales SympoAnforderungen an den Wasserbau,
sium 2008 Zürich, 11./12.9. 2008

DOBLER W (2006): Gravity current flow over submerged obstacles, Diplomarbeit am Institut für Wasserbau und Wasserwirtschaft der Technischen Universität Graz

FORD D, JOHNSON M (1983): An Assessment of Reservoir Density Currents and Inflow Processes, Vol. E-83-7, U.S. Army Engineer Waterways Experiment Station, Vicksburg, Mississippi

HABERSACK H M, NACHTNEBEL H P, SCHNEIDER J, BOGNER K, BRAUNER M, MARKHART G, KOHL B, BADURA K, BRICIA F, GAMERITH B, KOBOLTSCHNIG G, MAYR P, MISCHKER J, ÖHLBÖCK K, PREINSDORFER S, SANTNER P, WAKONIG B, BOTTHOF M und FIEFER S, SANTNER P, WAKONIG B, BOTTHOF M und FIE-
GER S (2002): Water resources management in a changing GER S (2002): Water resources management in a changing environment: the impact of sediment on sustainability, End-
bericht, Institut für Wasserwirtschaft, Hydrologie und konstruktivem Wasserbau, Universität für Bodenkultur, Wien
KOLLER-KREIMEL V (2008): Key facts and figures about HP issues, Austrian-French Workshop on Water Research and Policy Issues, Hydropower in the context of Research and Policy Issues, Hydropower in

\section{LANE-SERFF G F, BEAL L M, HADFIELD T D (1995):}

Gravity current flow over obstacles, J. Fluid Mech., vol. 292, pp. 39-53. Department of Oceanography, The University of Southhampton, SO17 1BJ, UK

MORRIS G L, FAN J (1998): Reservoir Sedimentation Handbook, McGraw-Hill Publishing Company

OEHY C (2003): Effects of obstacles and jets on reservoir sedimentation due ot turbidity currents. Laboratoire de Cone Constructions Hydraulique

ÖWAV (1998): Entleerung, Spülung und Räumung von Speichern und Becken. Schriftenreihe des Österreichischen Wasser- und Abfallwirtschaftsverbandes, Heft 117

PUTZ W (2007): Wirtschaftlichkeitsuntersuchung von Stauraumspülungen am Beispiel des Sölkspeichers, Diplomarbeit am Institut für Wasserbau und Wasserwirtschaft der Technischen Universität Graz

SCHNEIDER J (2002): Verlandungsproblematik eines alpinen Speichers am Beispiel des Sölkstaus, Dissertation an der Universität für Bodenkultur. Wien

SCHNEIDER J (2004): Durchschleusen von Dichteströmen als Alternative zu Stauraumspülungen zur Verringerung negativer Auswirkungen auf die Ökologie von Unterliegern, Symposium Lebensraum Fluss, Hochwasserschutz, Wasserkraft, Ökologie, 16.6.-19.6.2004, Wallgau, Deutschland

TROY W (2006): Naturmessungen zur Erfassung von Dichteströmen am Beispiel des Sölkstaus, Diplomarbeit am Institut für Wasserbau und Wasserwirtschaft der Technischen Universität Graz

WACHTER S (2008): Beschreibung von Dichteströmen im Sölkspeicher unter Anwendung von Naturmessungen Diplomarbeit am Institut für Wasserbau und Wasserwirtschaft der Technischen Universität Graz 